\title{
Whole-cell vaccine coated with recombinant calreticulin enhances activation of dendritic cells and induces tumour-specific immune responses
}

\author{
HONGYAN WU ${ }^{1}$, YU HAN $^{2}$, YE QIN $^{2}$, CHUNYU CAO $^{2}$, YAN XIA $^{2}$, CHANGBAI LIU $^{2}$ and YANLIN WANG ${ }^{1,2}$ \\ ${ }^{1}$ Department of Oncology, Zhongnan Hospital, Wuhan University, Wuhan, Hubei 430071; ${ }^{2}$ Institute of Molecular Biology, \\ Three Gorges University Medical College, Yichang, Hubei 443002, P.R. China
}

Received August 22, 2012; Accepted October 17, 2012

DOI: $10.3892 /$ or.2012.2142

\begin{abstract}
It has been reported that calreticulin (CRT) plays an important role in mediating immunogenic tumour cell death. In the process of tumour cell apoptosis induced by specific stimuli, CRT is quickly transferred from the endoplasmic reticulum to the cell membrane. As a specific ligand, CRT on the surface of apoptotic tumour cells could mediate the recognition and clearance of apoptotic tumour cells by professional and nonprofessional phagocytes. In our previous studies, we used B16-F1 mouse melanoma cells coated with mCRT-vGPCR (a recombinant fusion protein of mouse CRT and virus G-proteincoupled receptor) as a whole-cell tumour vaccine to immunise experimental animals and found that this whole-cell vaccine could strongly inhibit the growth of homologous tumours. In this study, we further evaluated immune responses induced by this mCRT-vGPCR-coated whole-cell vaccine both in vivo and in vitro. An in vitro phagocytosis assay showed that the mCRTvGPCR on the cell surface greatly enhanced the engulfment of B16-F1 cells by dendritic cells (DCs). The specific antitumour immune response was observed when the mCRT-vGPCRcoated B16-F1 cells were used as a whole-cell tumour vaccine to immune mice, which included significantly enhanced cytotoxic T lymphocyte (CTL) activities and increased the number of IFN- $\gamma$-producing $\mathrm{T}$ cells. These results indicate that the mCRT-vGPCR-coated whole-cell vaccine can induce specific antitumour immunity though the activation of DCs. These results may provide an experimental basis for the development of new tumour vaccines.
\end{abstract}

\section{Introduction}

Cancer affects a significant proportion of the population worldwide. Although numerous immune effector cells and molecules

Correspondence to: Professor Yanlin Wang, Department of Oncology, Zhongnan Hospital, Wuhan University, 185 Donghu Road, Wuhan, Hubei 430071, P.R. China

E-mail: fzswangyl@ctgu.edu.cn

Key words: calreticulin, whole-cell tumour vaccine, dendritic cell, tumour-specific immune response, immunogenic cell death are involved in the recognition and destruction of cancer cells by a process known as cancer immunosurveillance, the cancer cells can escape such monitoring through the production of poorly immunogenic tumour cell variants and the subversion of the immune system. Therefore, a method that can induce the immunogenic transformation of tumour cells and restore the body's immune response against tumour cells could represent an effective pathway for tumour prevention and therapy (1).

Whole-cell tumour vaccines have theoretical advantages over epitope-specific vaccines in that the former can present multiple and unknown tumour antigens to the immune system. However, since most types of tumour cells are poorly immunogenic in practise (2), improving the immunogenicity of the tumour vaccine is important for tumour-vaccine mediated immunotherapy.

Calreticulin (CRT) is a $46-\mathrm{kDa} \mathrm{Ca}^{2+}$-binding protein that is mainly located in the endoplasmic reticulum (ER). Previous studies have indicated that CRT is a multifunctional protein involved in a wide variety of cellular processes, including cell adhesion (3), the folding of newly synthesised glycoproteins (4-6), gene expression (4), $\mathrm{Ca}^{2+}$ homeostasis (7) and lectin-like chaperone activity (6). In addition to the ER, CRT has been found to be localised in various subcellular compartments, such as the cytosol, nucleus and cell surface membrane.

The CRT on the cell surface has multiple functions, including the modulation of cell adhesion and migration (8-11), and recent studies have found that the CRT on the cell surface also plays an important role in mediating the phagocytosis of apoptotic tumour cells by professional and non-professional phagocytes. During the process of tumour cell apoptosis, CRT is rapidly translocated from the ER to the cell surface and serves as an 'eat me' signal that can be recognised by the phagocytes within hours after the initiation of immunogenic cell death; at the same time, the tumour cells undergoing apoptosis down-regulate the expression of 'do not eat me' signals such as surface CD47 to facilitate the recognition and engulfment of the tumour cells by phagocytes and then to induce antitumour immune response (12). It has been reported that tumour cells killed by UVC, $\gamma$ irradiation (13) and some chemotherapeutics (14-17) could induce this type of tumour-specific immune response.

Since the CRT translocation from the ER to the cell surface is not an inherent feature of apoptosis in tumour cells but is only an event induced by specific stimuli in some tumour cell 
lines, a method that can easily coat CRT onto the surface of any tumour cell would aid in providing a potent research tool for antitumour immunotherapy. In our previous studies, we used a B16-F1 mouse melanoma cell line coated with mCRT-vGPCR as a whole-cell tumour vaccine to immunise experimental animals and found that this whole-cell vaccine could induce a strong antitumour effect against the homologous tumour (18). In this study, we further evaluated the immune responses induced by this mCRT-vGPCR-coated whole-cell vaccine both in vivo and in vitro.

\section{Materials and methods}

Mice and cell line. Female C57BL mice 5-6 weeks of age were purchased from the animal centre of Wuhan University. The animals were housed under specific pathogen-free conditions and the experiments were conducted in accordance with the ethical guidelines for the care and use of laboratory animals at Wuhan University. The mouse melanoma cell line B16-F1 was purchased from the Cell Bank of China (Wuhan). The cells were maintained at $37^{\circ} \mathrm{C}$ in $5 \% \mathrm{CO}_{2}$ and RPMI-1640 medium, which contains $10 \%$ heat-inactivated foetal bovine serum, $100 \mu \mathrm{g} / \mathrm{ml}$ streptomycin and $100 \mathrm{U} / \mathrm{ml}$ penicillin. The B16-F1 cell line with the mCRT-vGPCR high expression was constructed as previously reported (18).

Materials. Lipofectamine $2000^{\mathrm{TM}}$ transfection reagent and TRIzol reagent were obtained from Invitrogen (San Diego, CA, USA). Rabbit anti-human CRT polyclonal antibody was purchased from Stressgen (Victoria, BC, Canada). Rhodamine-labeled goat anti-rabbit IgG antibodies, mouse IFN- $\gamma$ ELISPOT kit and anti-mouse CD11c PE were purchase from eBioscience (San Diego, CA, USA). Recombinant murine IL-4 and GM-CSF were purchased from Peprotech (Rocky Hill, NJ, USA). CytoTox $96^{\circledR}$ Non-Radioactive Cytotoxicity assay was purchased from Promega (Madison, WI, USA). The RPMI-1640 cell culture medium and the other chemicals were from Sigma (St. Louis, MO, USA). BENS (Bis-ethylnorspermine) was kindly provided by Professor Robert A. Casero at Johns Hopkins University. All primer synthesis and DNA sequencing were performed by Sangon Biologic Engineering Technology \& Services Co. (Shanghai, China).

Detection of $m C R T-v G P C R$ mRNA by RT-PCR. When the mCRT-vGPCR-containing or empty plasmids were transfected into the B16-F1 cells, the total RNA was extracted from the stably transfected cells using the TRIzol reagent and then used to amplify mCRT-vGPCR or vGPCR mRNA by RT-PCR. The following were the PCR primers used in this experiment: for mCRT-vGPCR, upstream 5'-GATGGATGGAGAGTGGGA ACC-3'; downstream 5'-TCGATCTAGACTACCGCGATCGG TGCTTGCAAAA-3'. For vGPCR, upstream 5'-ATATCTCG AGATGGCGGCCGAGGATTTCCTAACC-3'; downstream 5'-TCGATCTAGACTACCGCGATCGGTGCTTGCAAAA-3'. For GAPDH (the internal control), upstream 5'-CAAGGTC ATCCATGACAACTTTG-3'; downstream 5'-GTCCACCACC CTGTTGCTGTAG-3'. The two primers for mCRT-vGPCR were located within the CRT and VGPCR portions, respectively. The length of the PCR product was $728 \mathrm{bp}$ for mCRT-vGPCR, $241 \mathrm{bp}$ for vGPCR and $496 \mathrm{bp}$ for GAPDH. The PCR products were separated on a $1.5 \%(\mathrm{w} / \mathrm{v})$ agarose gel, and the DNA was visualised by ethidium bromide staining.

Detection of $m C R T-v G P C R$ fusion protein by western blotting. The stably transfected cell lines were washed with PBS and lysed using Cymal-5 solution. The proteins in the lysate were separated by $10 \%$ SDS-PAGE and transferred onto a PVDF membrane. The membrane was blocked with fat-free milk solution $(5 \%, \mathrm{w} / \mathrm{v})$ overnight and then incubated with the rabbit anti-CRT polyclonal antibody (1:1000) at $4^{\circ} \mathrm{C}$ for $12 \mathrm{~h}$. After washing 3 times, the membrane was incubated with an HRP-conjugated anti-rabbit IgG antibody (1:4000) at room temperature for $1 \mathrm{~h}$ and developed using ECL.

Detection of mCRT-vGPCR expression by cell immunofluorescence $(C I F)$. The stably transfected cells were seeded in 24-well plates and cultured for $12 \mathrm{~h}$. After washing with PBS, the cells were fixed with $2 \%(\mathrm{~m} / \mathrm{v})$ paraformaldehyde (PFA) and incubated with $10 \%(\mathrm{v} / \mathrm{v})$ goat serum to block non-specific interactions. The cells were then incubated with a rabbit anti-CRT polyclonal antibody $(1: 200)$ at $4^{\circ} \mathrm{C}$ overnight. After washing 3 times with PBS, the cells were incubated with a rhodamine-conjugated goat anti-rabbit IgG polyclonal antibody (1:500) at room temperature for $1 \mathrm{~h}$ (in the dark). After washing 3 times with PBS, the cells were incubated with $300 \mathrm{nM}$ 4,6-diamidino-2-phenylindole (DAPI) for $10 \mathrm{~min}$ at room temperature (in the dark) to stain the nuclei. The samples were analysed using fluorescence microscopy (Nikon TE2000, Japan) with NIS-Elements BR 3.1 software (Nikon, Japan) to identify the mCRT-vGPCR fusion protein.

In vitro phagocytosis assay. Bone marrow (BM) cells were collected from the tibias and femurs of the C57BL mice using culture medium. Following centrifugation, the $\mathrm{BM}$ cells were resuspended in red-cell lysis solution $\left(0.15 \mathrm{M} \mathrm{NH}_{4} \mathrm{Cl}, 0.01 \mathrm{M}\right.$ $\mathrm{KHCO}_{3}$, and $1 \mathrm{mM}$ EDTA) for $1 \mathrm{~min}$ to remove the red blood cells. The BM cells were collected by centrifugation and cultured in a medium supplemented with $10 \mathrm{ng} / \mathrm{ml}$ recombinant mouse GM-CSF and $5 \mathrm{ng} / \mathrm{ml}$ recombinant mouse IL-4 in 6 -well plates $\left(1 \times 10^{6}\right.$ cells/well). After 7 days, the non-adherent and loosely adherent cells were harvested as the dendritic cells (DCs) and used as the effector cells for the phagocytosis assay. The stably transfected B16-F1 cells incubated with BENS for $48 \mathrm{~h}$ were labelled with the green dye CFDA-SE $(1 \mu \mathrm{M})$ for $20 \mathrm{~min}$ and used as the target cells. The effector and target cells were co-cultured at $37^{\circ} \mathrm{C}$ for $2 \mathrm{~h}$ at a $1: 1 \mathrm{E} / \mathrm{T}$ (effector/ target) ratio. After 3 washes, anti-mouse CD11c PE was added to the cell mixture for $30 \mathrm{~min}$ at room temperature (in the dark) to label the effector cells. The cells were washed with PBS and analysed by flow cytometry (FCM). The phagocytotic efficiency was represented by the cell ratio of the double-positive cell number over total cell number.

Whole-cell vaccine immunisation. The 7- to 8-week-old female C57BL mice were randomly divided into four groups: i) B16-mCRT-vGPCR ( $\mathrm{n}=10)$; ii) B16-vGPCR ( $\mathrm{n}=10)$; iii) B16 $(n=10)$, and iv) PBS $(n=10)$. In groups 1-3, the B16-F1 cells were treated by $10 \mu \mathrm{M}$ BENS for $48 \mathrm{~h}$ to induce cell apoptosis and then used as the whole-cell vaccine, subcutaneously inoculated into the back of each mouse. Instead of B16-F1 cells, PBS was 


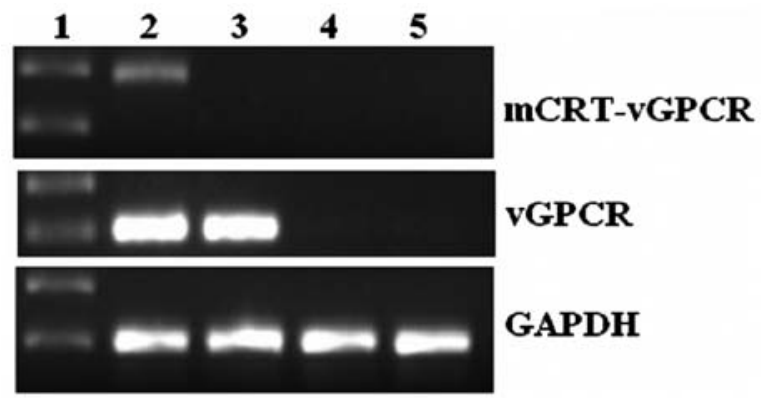

Figure 1. RT-PCR was used to determine the expression of mCRT-vGPCR at the mRNA level after pcDNA3.1(+)-mCRT-vGPCR was transfected into B16-F1 cells. Lane 1, DNA marker; lane 2, B16-F1 cells transfected with pcDNA3.1(+)-mCRT-vGPCR; lane 3, B16-F1 cells transfected with pcDNA3.1(+)-vGPCR; lane 4, B16-F1 cells transfected with pcDNA3.1(+); lane 5, B16-F1 control cells.

used to immune the mice in group 4. The whole-cell vaccine or PBS was injected 3 times at Days 0,10 and 20, and the mice were sacrificed 10 days after the last injection. The spleens from the vaccinated mice were collected for analysis.

Determination of specific cytotoxic T lymphocyte (CTL) activity. The splenocytes from the immunised mice were suspended in complete RPMI-1640 medium with $10 \%$ fetal calf serum and used as the effector cells to assay their specific cytotoxic T lymphocyte (CTL) activity. B16-F1 cells were used as the target cells in this experiment. The effector cells $\left(5 \times 10^{4}\right.$ $2 \times 10^{5}$ cells/well) were stimulated with IL-2 before use and incubated with the target cells $\left(1 \times 10^{4}\right.$ cells/well $)$ at E:T ratios of 20:1, 10:1, and 5:1 in a total volume of $200 \mu \mathrm{l}$ RPMI-1640 medium. The released lactate dehydrogenase (LDH) was measured according to the manufacturer's instructions after $4 \mathrm{~h}$ of incubation at $37^{\circ} \mathrm{C}$ in $5 \% \mathrm{CO}_{2}$. The percentage of specific killing was calculated as follows: specific killing \% $=($ experimental release - spontaneous release $) /($ total release spontaneous release).

Enzyme-linked immunospot (ELISPOT) assay. The number of splenocytes that could produce interferon- $\gamma($ IFN- $\gamma$ ) was quantified by the cytokine-specific enzyme-linked immunospot (ELISPOT) assay. Plates (96 wells) were coated with $100 \mu \mathrm{g}$ of anti-mouse IFN- $\gamma \mathrm{McAb}$ overnight at $4^{\circ} \mathrm{C}$, washed with PBS and blocked for $1 \mathrm{~h}$ with $5 \%$ BSA. The splenocytes were seeded into each well $\left(2 \times 10^{5}\right.$ cells/well) and cultured for $24 \mathrm{~h}$ in RPMI-1640 alone (negative control) or with the apoptotic B16-F1 cells induced by BENS or $10 \mu \mathrm{g} / \mathrm{ml}$ concanavalin A (positive control). After washing with PBS, followed by PBS- $0.05 \%$ Tween-20, the biotinylated rabbit anti-IFN- $\gamma$ antibody was added and incubated for $1 \mathrm{~h}$ at room temperature. The plates were washed in PBS-0.05\% Tween-20, and then avidin-HRP solution was added and incubated for $45 \mathrm{~min}$ at room temperature. After rewashing, the cells were treated with $100 \mu$ l of freshly prepared AEC substrate solution and incubated at room temperature in the dark to monitor the development of spots. The reaction was stopped by washing 3 times with $200 \mu \mathrm{l} /$ well distilled water. The plate was air-dried, and the spots were counted using an automated ELISPOT plate reader.

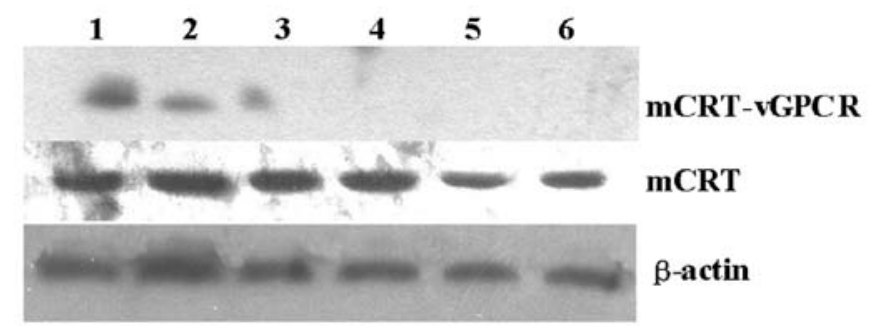

Figure 2. Western blotting was used to identify the expression of mCRTvGPCR in B16-F1 cells. Lanes 1 and 2, B16-F1 cell lines transfected with pcDNA3.1(+)-mCRT-vGPCR; lane 3, mixed B16-F1 cells transfected with pcDNA3.1(+)-mCRT-vGPCR; lane 4, B16-F1 cell line transfected with pcDNA3.1(+)-vGPCR; lane 5, B16-F1 cell line transfected with pcDNA3.1(+); lane $6, \mathrm{~B} 16-\mathrm{F} 1$ control cells.

Statistical analysis. Student's t-tests were used for the comparison of the results between the different groups. The tests were performed using SPSS software. $\mathrm{P}<0.05$ was considered to indicate statistically significant differences.

\section{Results}

Expression of $m C R T-v G P C R$ in B16-F1 cells. When the mCRT-vGPCR-containing or empty plasmids were transfected into the B16-F1 cells, a high level of mCRT-vGPCR mRNA was observed only in the mCRT-vGPCR-transfected cell line by RT-PCR (Fig. 1). As the two primers used for mCRT-vGPCR in the PCR reactions were located in the mCRT and vGPCR regions, respectively, only the mRNA from the fusion gene could be used as the template for the PCR. Using western blot analysis with an anti-CRT antibody, the mCRT-vGPCR fusion protein was also detected only in the mCRT-vGPCR- transfected cells. As shown in Fig. 2, the mCRT-vGPCR fusion protein has a higher molecular weight (110 kDa) than the native CRT protein $(55 \mathrm{kDa})$.

A CIF analysis was used to further determine whether the mCRT-vGPCR fusion protein could be localised to the cell surface. The two B16-F1 cell lines positively transfected with pcDNA3.1(+)-mCRT-vGPCR showed significant membrane expression of mCRT-vGPCR (Fig. 3), indicating that the mCRT-vGPCR fusion protein was efficiently expressed on the cell surface of the B16-F1 tumour cells.

$m C R T-v G P C R$ on the cell surface enhances the phagocytosis of B16-F1 cells by DCs in vitro. In view of the established role of CRT as an 'eat me' signal, we further investigated the possible effect of mCRT-vGPCR on the phagocytosis of BENStreated B16-mCRT-vGPCR cells by DCs, which are among the most efficient antigen-presenting cells. The labelled-DCs from C57BL mice (the effector cells) and transfected B16-F1 cells (the target cells) were co-cultured for $2 \mathrm{~h}$ in a 1:1 effector/target ratio, and FCM was used for the analysis. The results showed that, compared to the B16-F1 cells transfected with the empty vector, $>5$-fold higher phagocytotic efficiency was observed when the B16-F1 cells transfected with pcDNA3.1(+)-mCRTvGPCR were used as the target cells, indicating that the mCRT-vGPCR on the cell surface enhanced the phagocytosis of the tumour cells by the DCs (Fig. 4). 


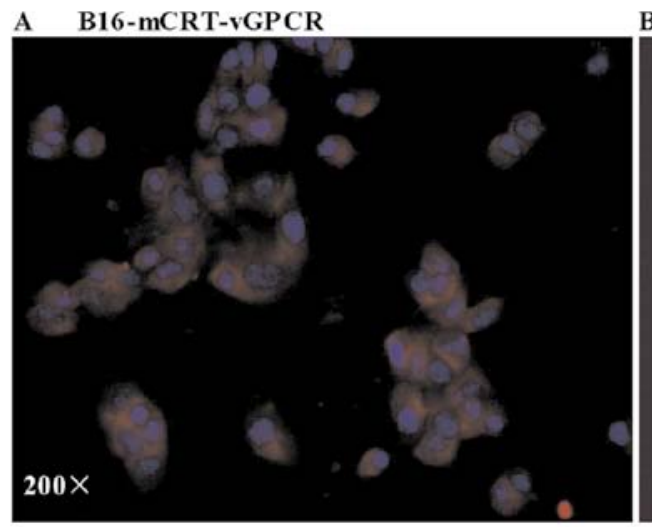

B Primary antibody free
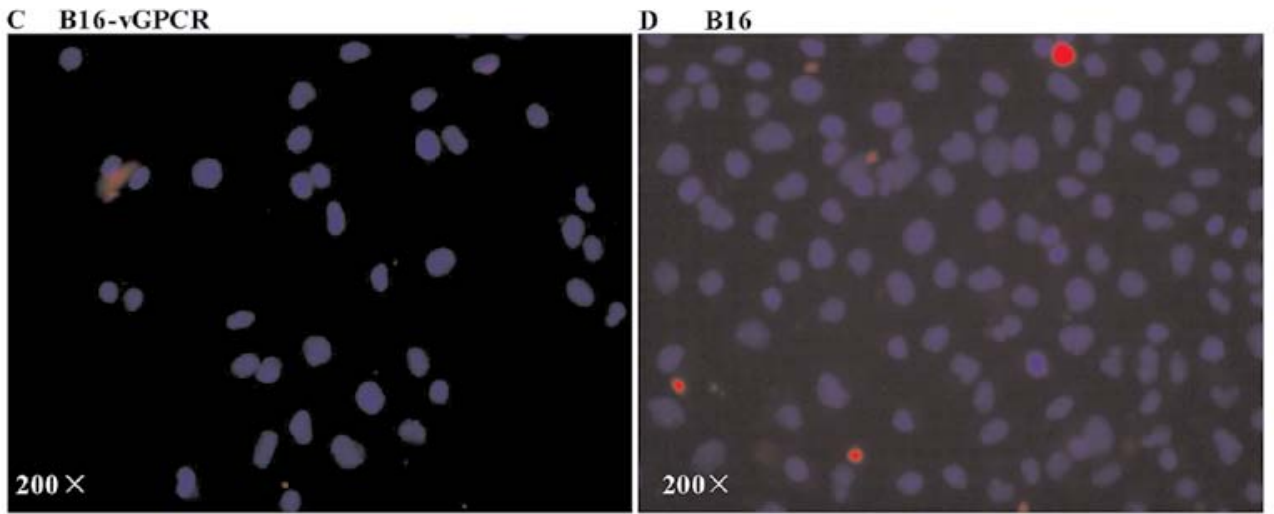

Figure 3. Immunofluorescence was used to identify the subcellular localisation of the mCRT-vGPCR fusion protein in B16-F1 cells. The red colour represents the CRT recognised by the rabbit anti-CRT antibody. The blue colour indicates the nuclei stained with DAPI. (A) B16-F1 cells transfected with pcDNA3.1(+)mCRT-vGPCR; (B) B16-F1 control cells without primary antibody; (C) B16-F1 cells transfected with pcDNA3.1(+)-vGPCR; and (D) B16-F1 control cells.

The $m C R T$-vGPCR-coated whole-cell vaccine significantly induces specific CTL activity in mice. The splenocytes from the immunised mice were used as the expanded effector (E) cells, and the B16-F1 cells were used as the target (T) cells in this experiment. The effector cells were stimulated by the BENStreated B16-F1 cells in vitro before use and then incubated with the target cells at E:T ratios of 20:1, 10:1, and 5:1 for $4 \mathrm{~h}$. The specific CTL activities were determined using the LDH assay. The results showed that the CTL activities were enhanced in the mice immunised with the BENS-treated B16-mCRT-vGPCR cells compared to the other groups $(\mathrm{P}<0.01)$ (Fig. 5).

Cytokine secretion assay. To examine whether Th1 or Th2 responses occurred in each immunised group, the IFN- $\gamma$ producing cells in the splenocytes from the immunised-mice groups were examined using the ELISPOT technique. The results showed that the immunisation with the BENS-treated B16-mCRT-vGPCR cell vaccine elicited a significantly higher number of IFN- $\gamma$-producing cells in the splenocytes (Fig. 6). Since IFN- $\gamma$ is an indicator of the Th1-based response, our data suggested that the mCRT-vGPCR- coated cell vaccine mainly induced Th1-based immune responses.

\section{Discussion}

Whole-cell tumour vaccines have been investigated for more than 20 years in both preclinical models and clinical trials in humans (19-21). The advantage of whole-cell vaccinations over other types of immunotherapy that target specific antigens is that multiple and unknown tumour antigens may be targeted by both the innate and adaptive immune systems. However, the whole-cell vaccines have not resulted in significant longterm therapeutic benefits $(22,23)$. One possible explanation is that only a small proportion of the molecules expressed on the cell surface are specific for cancer cells, whereas the vast majority of cell surface components are derived from housekeeping genes, carbohydrates, lipids and other molecules that are ubiquitously expressed by normal cells (24). As a result, the immune response induced by whole-tumour cell vaccine is insufficient to eliminate the cancer cells in vivo. Therefore, further improvements are necessary to increase the immunogenicity of cancer cell-based vaccines.

Recent studies have identified a new CRT-mediated mechanism for tumour cell immunogenic death $(12,13,25)$. CRT is an ER resident chaperone protein with multiple physiological functions and can rapidly translocate to the cell surface of tumour cells during the apoptosis induced by specific stimuli, such as anthracycline treatment. When these apoptotic tumour cells with CRT coated on the surface were used as the vaccines to immune mice, the specific antitumour immune response was induced.

As the translocation of CRT from the ER to the cell surface is not an inherent feature of apoptosis in tumour cells, a method that can easily coat CRT onto the surface of tumour cells would provide a potent research tool for antitumour immunotherapy. In our previous studies, an mCRT-vGPCR recombinant gene 

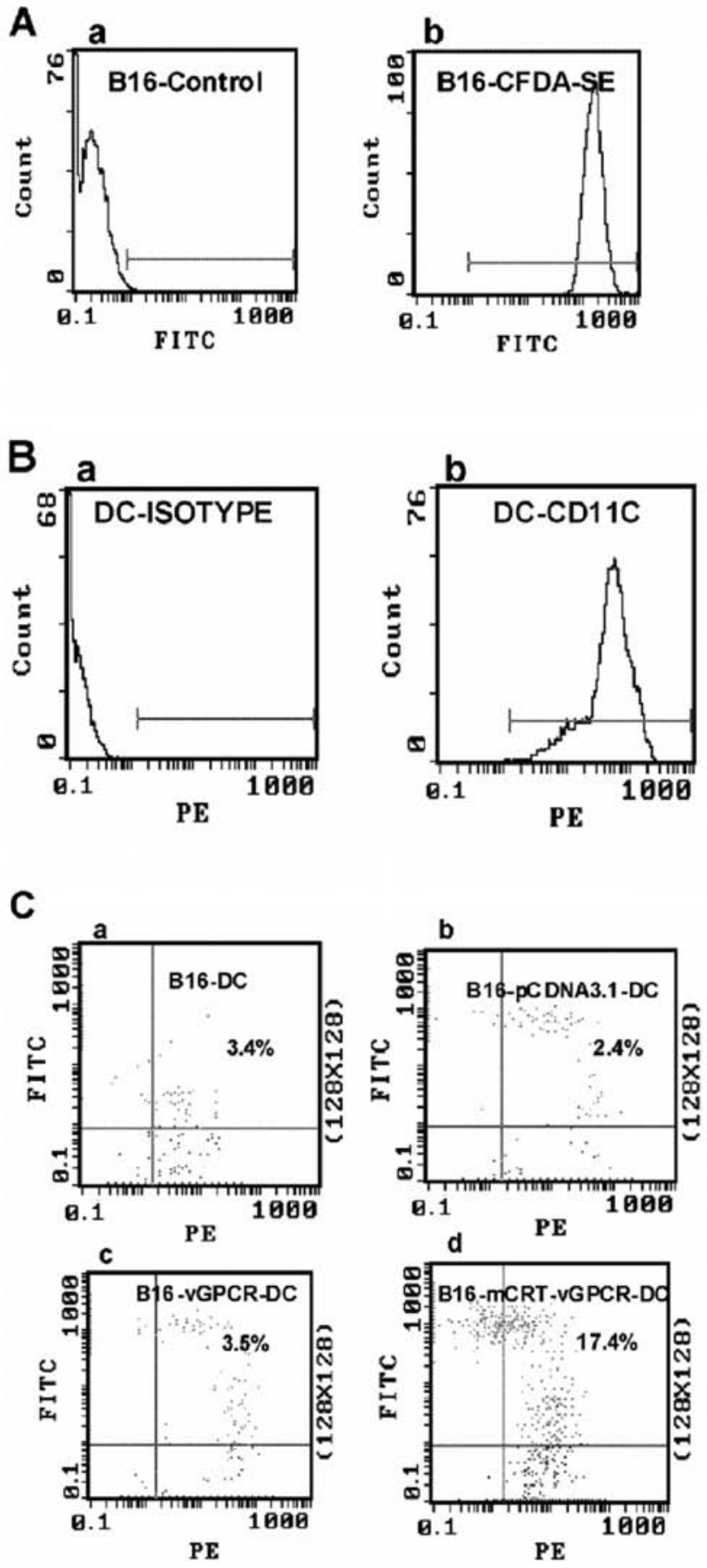

Figure 4. The mCRT-vGPCR fusion protein on the cell surface enhanced the phagocytosis of B16-F1 cells by DCs in vitro. (A) Target cells labelled with green dye CFDA-SE; (a) B16-F1 control cells, (b) B16-F1 cells stained with CFDA-SE. (B) Labelled DCs with PE-coupled anti-CD11c polyclonal antibody; (a) DC-isotype, (b) DCs labelled with PE-coupled CD11c antibody. (C) mCRT-vGPCR on the cell surface enhanced the phagocytosis of B16-F1 cells by DCs in vitro; (a) B16-F1 cells were used as the target cells, (b) B16-F1 cells transfected with pCDNA3.1 were used as the target cells, (c) B16-F1 cells transfected with pCDNA3.1-vGPCR were used as the target cells, and (d) B16-F1 cells transfected with pcDNA3.1(+)-mCRT-vGPCR were used as the target cells.

was constructed and stably transfected into mouse melanoma B16-F1 cells. vGPCR is a membrane protein that contains seven transmembrane domains, with the N-terminus protruding extracellularly (26). Therefore, the mCRT-vGPCR fusion protein

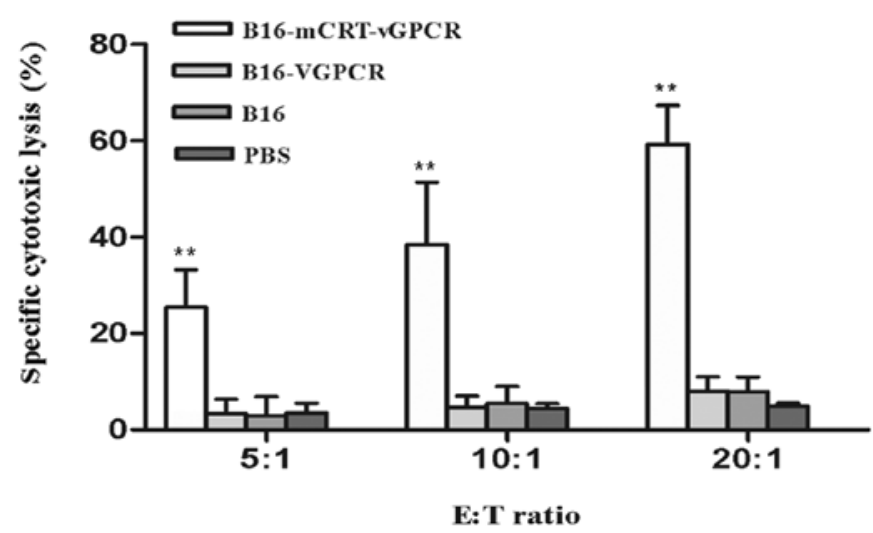

Figure 5. Enhanced specific CTL activity in the splenocytes from the mice immunised with the BENS-treated B16-mCRT-vGPCR whole-cell vaccine. The splenocytes from the immunised mice were used as the effecter cells (E), and the B16-F1 cells were used as the target cells (T) at E:T ratios of 20:1, 10:1, and 5:1. The data are presented as $X \pm S D(n=10) .{ }^{* *} P<0.01$ compared with the PBS group.

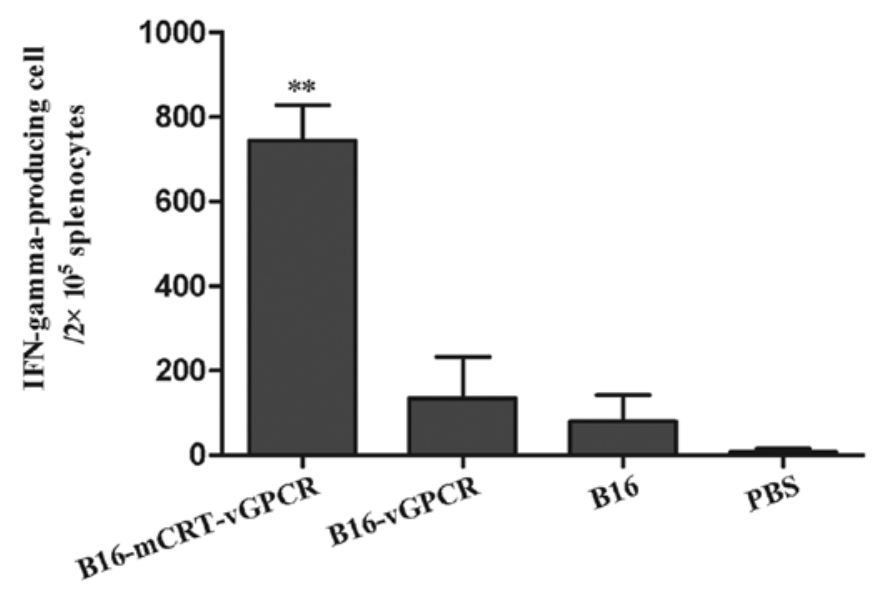

Figure 6. The ELISPOT method was used to assay the IFN- $\gamma$-producing cell number. The data represent the means of the spot-forming cell number per $2 \times 10^{6}$ splenocytes. ${ }^{* *} \mathrm{P}<0.01$ compared with the PBS group.

was efficiently expressed on the cell surface via the ability of vGPCR to localise to the membrane. When this B16-F1 cell line coated with mCRT-vGPCR as a whole-cell vaccine to immunise the mice, we found that this whole-cell vaccine could strongly inhibit the growth of homologous tumour. In this study, we further evaluated the immune responses induced by this mCRTvGPCR-coated whole-cell vaccine both in vivo and in vitro.

DCs play important roles in processing and presenting antigens, thus the recognition and phagocytosis of a tumour cell vaccine by DCs is a key event in whole-cell tumour vaccine-mediated immunotherapy. In this study, we found that, similar to the native CRT, the mCRT-vGPCR on the tumour cell surface could also mediate the phagocytosis of the tumour cells by DCs. Compared with the B16-F1 cells transfected with the empty vector, more than 5-fold higher phagocytotic efficiency was observed when the B16-F1 cells transfected with pcDNA3.1(+)- mCRT-vGPCR were used as the target cells.

Subsequently, these mCRT-vGPCR-coated B16-F1 cells were treated by polyamine analogue BENS to induce cell 
apoptosis and then used as the whole-cell vaccine to immunise C57BL mice. We chose BENS as an apoptosis-inducing agent as it has no effect on the subcellular localisation of CRT (27). The results indicated that the mCRT-vGPCR-coated whole-cell vaccine could significantly induce specific CTL activity and IFN- $\gamma$ secretion of the splenocytes, both of which are important indicators of the specific immune responses stimulated by the vaccine in the model animals.

In conclusion, the mCRT-vGPCR-coated whole-cell vaccine can induce specific antitumour immunity and these results may provide an experimental basis for the development of new tumour vaccines.

\section{Acknowledgements}

This study was supported by a grant from the National Natural Science Foundation of China (no. 30973445), the Fundamental Research Funds for the Central Universities, the Research Foundation of Outstanding Young Innovative Team Projects of Hubei Provence (T201203).

\section{References}

1. Zitvogel L, Tesniere A and Kroemer G: Cancer despite immunosurveillance: immunoselection and immunosubversion. Nat Rev Immunol 6: 715-727, 2006.

2. Huang X, Ye D and Thorpe PE: Enhancing the potency of a whole-cell breast cancer vaccine in mice with an antibody-IL-2 immunocytokine that targets exposed phosphatidylserine. Vaccine 29: 4785-4793, 2011.

3. Johnson S, Michalak M, Opas M and Eggleton P: The ins and outs of calreticulin: from the ER lumen to the extracellular space. Trends Cell Biol 11: 122-129, 2001.

4. Michalak M, Corbett EF, Mesaeli N, et al: Calreticulin: one protein, one gene, many functions. Biochem J 344: 281-292, 1999.

5. Trombetta ES and Parodi AJ: Quality control and protein folding in the secretory pathway. Annu Rev Cell Dev Biol 19: 649-676, 2003.

6. Trombetta ES: The contribution of $N$-glycans and their processing in the endoplasmic reticulum to glycoprotein biosynthesis. Glycobiology 13: R77-R91, 2003.

7. Michalak M, Robert Parker JM and Opas M: $\mathrm{Ca}^{2+}$ signaling and calcium binding chaperones of the endoplasmic reticulum. Cell Calcium 32: 269-278, 2002.

8. Zhu Q, Zelinka P, White T and Tanzer ML: Calreticulin-integrin bidirectional signaling complex. Biochem Biophys Res Commun 232: 354-358, 1997.

9. Kwon MS, Park CS, Choi K, et al: Calreticulin couples calcium release and calcium influx in integrin-mediated calcium signaling. Mol Biol Cell 11: 1433-1443, 2000.
10. Gray AJ, Park PW, Broekelmann TJ, et al: The mitogenic effects of the B-chain of fibrinogen are mediated through cell surface calreticulin. J Biol Chem 270: 26602-26606, 1995.

11. White TK, Zhu Q and Tanzer ML: Cell surface calreticulin is a putative mannoside lectin which triggers mouse melanoma cell spreading. J Biol Chem 270: 15926-15929, 1995.

12. Obeid M, Tesniere A, Ghiringhelli F, et al: Calreticulin exposure dictates the immunogenicity of cancer cell death. Nat Med 13: 54-61, 2007.

13. Obeid M, Panaretakis T, Joza N, et al: Calreticulin exposure is required for the immunogenicity of gamma irradiation and UVC light-induced apoptosis. Cell Death Differ 14: 1848-1850, 2007.

14. Spisek R and Dhodapkar MV: Towards a better way to die with chemotherapy: role of heat shock protein exposure on dying tumor cells. Cell Cycle 6: 1962-1965, 2007.

15. Spisek R, Charalambous A, Mazumder A, Vesole DH, Jagannath S and Dhodapkar MV: Bortezomib enhances dendritic cell (DC)-mediated induction of immunity to human myeloma via exposure of cell surface heat shock protein 90 on dying tumor cells: therapeutic implications. Blood 109: 4839-4845, 2007.

16. Tesniere A, Panaretakis T, Kepp O, et al: Molecular characteristics of immunogenic cancer cell death. Cell Death Differ 15: $3-12,2008$

17. Panaretakis T, Joza N, Modjtahedi N, et al: The co-translocation of ERp57 and calreticulin determines the immunogenicity of cell death. Cell Death Differ 15: 1499-1509, 2008.

18. Qin Y, Han Y, Cao C, Ren Y, Li C and Wang Y: Melanoma B16-F1 cells coated with fusion protein of mouse calreticulin and virus G-protein coupled receptor induced the antitumor immune response in Balb/C mice. Cancer Biol Ther 11: 574-580, 2011.

19. Copier J and Dalgleish A: Overview of tumor cell-based vaccines. Int Rev Immunol 25: 297-319, 2006.

20. de Gruijl TD, van den Eertwegh AJ, Pinedo HM and Scheper RJ: Whole-cell cancer vaccination: from autologous to allogeneic tumor- and dendritic cell-based vaccines. Cancer Immunol Immunother 57: 1569-1577, 2008.

21. Chiang CL, Benencia $F$ and Coukos G: Whole tumor antigen vaccines. Semin Immunol 22: 132-143, 2010.

22. Copier J and Dalgleish A: Whole-cell vaccines: a failure or a success waiting to happen? Curr Opin Mol Ther 12: 14-20, 2010.

23. Bodey B, Bodey B Jr, Siegel SE and Kaiser HE: Failure of cancer vaccines: the significant limitations of this approach to immunotherapy. Anticancer Res 20: 2665-2676, 2000.

24. Cohen EP, Chopra A, O-Sullivan I and Kim TS: Enhancing cellular cancer vaccines. Immunotherapy 1: 495-504, 2009.

25. Fucikova J, Kralikova P, Fialova A, et al: Human tumor cells killed by anthracyclines induce a tumor-specific immune response. Cancer Res 71: 4821-4833, 2011.

26. Liu C, Sandford G, Fei G and Nicholas J: G protein selectivity determinant specified by a viral chemokine receptor-conserved region in the $\mathrm{C}$ tail of the human herpes virus $8 \mathrm{G}$ protein-coupled receptor. J Virol 78: 2460-2471, 2004.

27. Cao CY, Han Y, Ren YS and Wang YL: Apoptotic B16-F1 cells coated with recombinant calreticulin mediated anti-tumor immune response in mice. Chin J Cancer Res 22: 253-259, 2010. 